Article

\title{
Full IoT Lora School Building Management System
}

\author{
Bruno Mataloto ${ }^{1}$, Joao C Ferreira ${ }^{1,2, *}$ and Nuno Cruz ${ }^{3}$ \\ 1 Instituto Universitário de Lisboa (ISCTE-IUL), ISTAR-IUL, Portugal; \{Bruno_Mataloto,jcafa\}@iscte-iul.pt \\ 2 INOV INESC Inovação - - Instituto de Novas Tecnologias; joao.ferreira@inov.pt \\ 3 ISEL/IPL \& LASIGE/FC/UL, Portugal, ncruz@isel.ipl.pt \\ * Correspondence: jcafa@iscte-iul.pt; Tel.: Tel.: +351-210-464-277 (J.C.F.)
}

\begin{abstract}
In this research paper we describe the development phase of a low-cost LoRa IoT solution applied to a kindergarten school with three years results. A set of sensors solution was developed in a LoRa communication board, battery powered, providing a simplified setup process. These sensors were used in order to measure temperature, humidity, luminosity, air quality and presence. Also, energy monitor solutions were integrated. The acquired data is transmitted and analysed for knowledge extraction, identifying savings and other related KPIs. From data, automatic saving actions were performed towards heating and cooling systems, lighting and a set of if-then actions were developed for automatic cost-saving actions, based on infrared signals to heating/cooling systems using some procedure of external command devices. This approach avoids the usage of proprietary vendor solutions in a flexible approach that can easily be deployed to any building facility. This is an important achievement since most of the building consumption is based on heating and cooling systems. In a three years test of the solution, the total energy consumption savings surpassed $20 \%$.
\end{abstract}

Keywords: IoT, LoRa, sustainability, building management system, sensors,

\section{Introduction}

Energy consumption in buildings is responsible for thermal comfort, climate, lighting, communication and entertainment. This consumption represents nowadays of $40 \%$ of total energy consumption sanitation and hygiene, and nutrition, as well as other amenities - are responsible for a significant share of energy use worldwide. Buildings are one of the major sources of energy consumptions and the ratio of this energy against total energy consumption increased from $34 \%$ to $41 \%$ in 30 years (1980 to 2010) [1].

The ratio of building energy consumption to total energy consumption increased from $33.7 \%$ to 41.1\% between 1980 and 2010 in the U.S.[2]. Higher Education institution campuses can utilise IoT related technologies in order to enhance connectivity and introduce automation, thereby reducing energy consumption costs campus-wide improving energy efficiency at classrooms with intelligent control of climate, lighting and also wasted energy.

Several trials have already been performed: 1) New Richmond schools in Tipp City, Ohio saved approximately $\$ 128,000$ each year by using a web-based system that controls all mechanical equipment inside its buildings [3]. Also saving in water can be performed with control of loses and automation for garden watering systems [4].

Nowadays building management became a reality with easy installation of sensors with batteries (no cables or associated power issues), allowing the deployment of solutions to control energy consumption and identify actions that would introduce savings and consequently reduce costs.

IoT economy is growing in all areas with the increasing availability of sensors at low costs, with the introduction of new transmission solutions to save sensor power and IoT platforms to store, 
visualise and manipulate the data. This will be a market of $\$ 6.3$ trillion by 2025 , according to McKinsey Global [5].

Today building management systems are expensive, with costs of $\$ 2.5$ to $\$ 7$ per square meter [6]. Despite these prices, the investment is paid due to savings created by IoT solutions that also introduce savings on labour costs because of the introduction of automated actions.

Traditional Building Management Systems (BMS) are based on pre-defined rules created by developers or administrators who are less effective when compared with IoT context-aware solutions, where systems can react to local measurements, and perform data collection based on external temperature, number of persons in a room and others information such as thermal performance of the building material.

Regarding communications, LoRaWAN fulfils the IoT requirements providing a bi-directional communication end to end in a long-range low power. LoRaWAN is available in more than 500 countries, and big companies like Cisco are providing commercial solutions. There are three types of LoRaWAN devices: 1) Class A, mandatory for all devices, devices that wake up only specific time to send data to save power and support bidirectional communication, 2) Class B that use extra receive windows during downlink period in addition to two time slots specified in class-A; and 3) Class $\mathrm{C}$ devices that are always listening. They can work as emergency alerts or automation controls with the lowest latency on the downlink. Also, LoRa performs better in buildings due to the ability of penetration on walls with less interference compared with others like Wi-Fi, Bluetooth, ZigBee, and Z-Wave. Also, more than 80 million LoRa connected devices are already introduced in more than 100 countries and with a CAGR for $100 \%$ or double each year ${ }^{1}$.

Considering this scenario and based on previous works [7,8], we developed a low-cost LoRa solution to monitor building environment and reduce costs. Our work is also being applied in a university campus and a data centre. Besides the creation of an easy installation scenario (no power feeding cables involved), an open-source visualization and alerts platform was developed to collect and show sensor data using a LoRa communication. Interactions with heating/cooling systems are available through an infrared solution, already in place.

Energy management in the context of the building is a similar system to our proposal, but traditionally these Energy Management System (EMS) are based on Smart devices, Wireless Sensor Networks (WSN), and a centralized platform for data presentation and management. Example of this systems is [9-11], an implementation of an EMS a Wireless Sensor Network using a ZigBee Module for communication with sensor nodes. Han et al. in [12] introduced a system for monitoring power consumption using ZigBee as the communication protocol in a WSN. Other works have been extended to wider ranges in the IoT paradigm utilizing the GSM/GPRS networks to remotely control the end-devices in [13-14], but these networks have the problem of power consumption, so this is not the right approach for battery-powered sensors that have the advantage of avoiding installation cables. Other studies applied scheduling approaches to optimize HVAC energy consumption [15-17]. In [18], a home controller system interfaced with device sensors is responsible for aggregated energy reporting of all devices to homeowners. In [19] authors create an IoT based DC powered homes to develop a DC distribution system encompassing all residential DC-based loads that interact with each other. However, the lack of standardized protocols and regulations were the main challenges in considering intelligent DC powered homes a suitable replacement to AC power systems. Challenges could be overcome with IoT that will provide an integrated platform for DC powered technologies inefficient energy distribution.

Using smart meter data, this EMS can monitor and provide real-time information on home energy consumption along with online access to devices status, thus allowing remote control of devices by customers. The proposed design is based on standard HTTP protocol and does not provide support for lighter-weight communication protocol like MQTT which is essential to scale up the system in order to accommodate multiple residential areas. In [20], a residential gateway controller was developed with a central management system that generated an operation plan for all the connected nodes in a home network depending on weather conditions.

${ }^{1}$ https://inbuildingtech.com/smart-buildings/lora-iot-inbuilding-connectivity/ 
Additionally, the researchers in [21] propose a cost modelling scheme for an optimization based energy management model that aims at reducing energy expenses of consumers. Other study found that Home energy management system can reduce electricity consumption by $15 \%$ potentially with acceptable discomfort [22].

\section{Platform Proposal}

As technology progresses and people get aware of all the new possibilities for wellbeing improvement, energy savings and resource management, local companies try to invest and obtain visible results. "Pequenos Sorrisos, Lda.", a kindergarten with 50 students and 12 rooms of 16 to 40 $\mathrm{m}^{2}$ located at Amadora, Lisbon, Portugal, has been working on a sustainability project since 2016, which includes a real time energy monitoring platform available on the market, total information digitalization and total lighting systems upgrade to LEDs. The energy monitoring platform consists of 3 amperometric clamps placed on each phase at the main circuit board that transmits all the data to a cloud infra-structure, where the dashboard provided with the product displays real time, hourly, daily and monthly consumptions.

Some of the detected problems were related to standby consumptions, however, the main part of the energy bill was due to kitchen appliances and the Air Conditioning system.

As dealing with standby energy consumption was unavoidable, and replacing kitchen appliances would introduce large costs, because managing the Air Conditioning system is very time consuming and reliant on staff behavior, the owners agreed to integrate a LoRa intelligent automation system to monitor each room's conditions and interact with the Air Conditioning system, the lighting, (which was often unintentionally left on), and smart WiFi sockets, based on the collected and processed information.

The proposed system can be structured in 3 separated layers, the Physical layer with the sensors hub and actuators, the Network layer with all the IP and LoRa communications and finally the Application layer where all the data is processed and displayed.

\subsection{Physical Layer}

The developed board (Figure 1), consists of 4 different types of sensors, temperature/humidity with a DHT22 sensor, light with a photoresistor sensor, presence with a PIR sensor and air quality with a MQ-135 sensor suitable for detecting of NH3, NOx, alcohol, Benzene, smoke, CO2, and others, all connected to a microcontroller powered by a simple Lithium Polymer battery with a capacity of $2400 \mathrm{mAh}$ and a small solar panel to restore is power over time.

The Raspberry Pi, (Figure 2) is the core of the system, responsible for LoRa network management, data processing and storage.

The energy system, (Figure 3) is an ongoing solution that will be integrated into the system.

Smart wall switches, (Figure 4) work as an interface between the ceiling lights and WiFi, and the same with smart sockets, (Figure 5) and appliances, allowing their control over the Internet and therefore the automation system.

A WiFi controlled device that emits infra-red signals, (Figure 6) will be used to send commands to Air Conditioning units, mirroring the controller itself. This device captures Infrared signals and stores them as commands. Each command works as the AC remote buttons, allowing the system to change each parameter independently such as fan speed, temperature, or working mode (winter/summer/dehumidifier). 


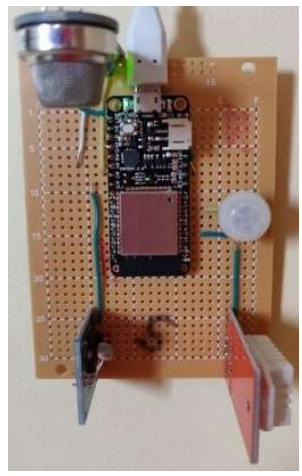

Figure 1-Developed sensor board

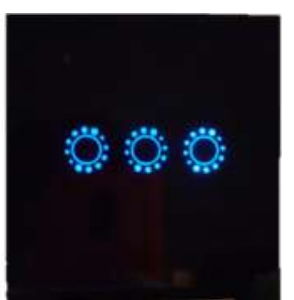

Figure 4- Smart wall switch

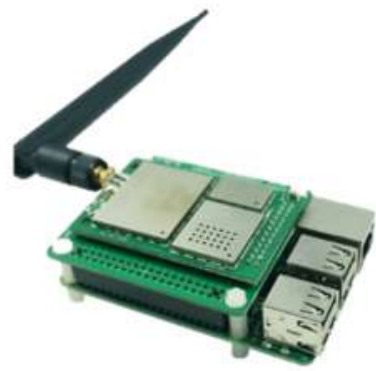

Figure 2- Raspberry Pi with LoRa Hat

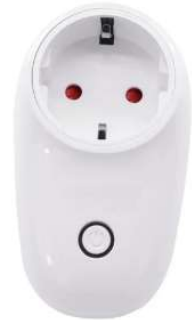

Figure 5-Smart power socket

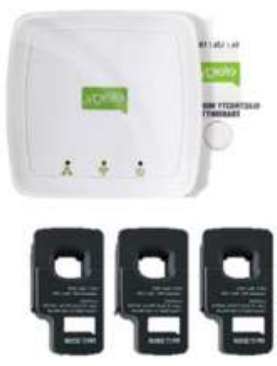

Figure 3- Energy monitoring system

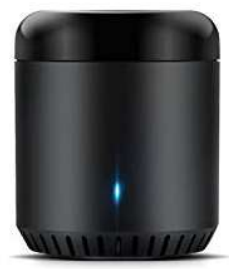

Figure 6- WIFI / Infra-Red Emitter

\section{$2.2 \quad$ Network Layer}

The entire system depends on 3 wireless communications protocols, LoRaWAN, WiFi and IR:

The LoRaWAN protocol is used to establish a connection between the sensors and the LoRa gateway running on the Raspberry Pi.

As a member of the LoRa Alliance, The Things Network, or TTN, is a worldwide project focused on developing open access and secure IoT network based on LoRa technology and LoRaWAN protocol. One of TTN's best feature is the possibility of creating a homemade Gateway with relatively cheap and easy to acquire hardware. The software setup is fully documented and, in a few hours, the Gateway is ready to receive messages sent from the sensors.

Each sensor is added to the network using the TTN console that provides a user-friendly interface to make device management as easy as possible. It also allows the integration of a variety of services such as HTTP, however, the Application server that will be used is going to connect directly to TTN.

$\mathrm{TCP} / \mathrm{IP}$ over WiFi will connect all the other devices, actuators and energy monitoring system to the Raspberry Pi where HTTP requests will be originated to change devices states.

If This Than That, or IFTTT is a free Web platform that allows the interaction between services and dozens of different APIs provided by many manufacturers all around the world, like weather service, time service, HTTP service, voice assistant APIs or smart devices API's.

As both IR emitters, WiFi switches and power sockets require interaction with their API to be remotely controlled, this is done using IFTTT HTTP service, and all notifications are also sent using IFTTT email services.

A schematic of the project communication network is shown in Figure 7. 


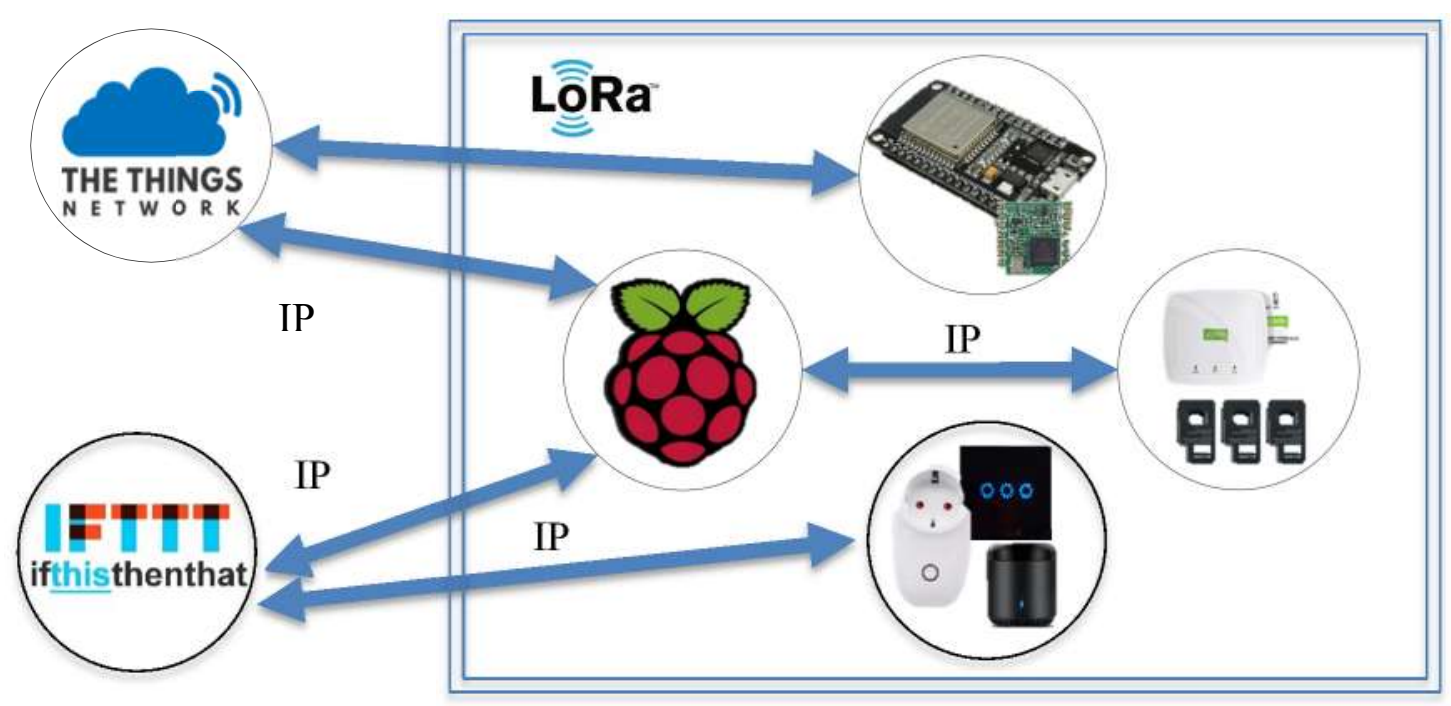

Figure 7 - System network schematic

\subsection{Application Layer}

The Raspberry Pi is where services and applications run, these include instances of Node-RED and Maria-DB.

Node-RED is an open source software available for many platforms including the Raspberry Pi. It is a flow-based programming software, created specifically for IoT applications where it is possible to connect API's, physical devices, other network interfaces and web services. It also allows the programming of rules in JavaScript to run or keep from running specific flows or the installation of extra features such as a TTN node that provides connectivity to TTN. Node-RED will also connect to a local MySQL database where all the data will be stored. Temperature, humidity, light, presence, air quality values and other variables for each classroom will be acquired and stored locally and will allow data extraction at a later stage. A schematic of how the most important applications interact is presented in Figure 8.

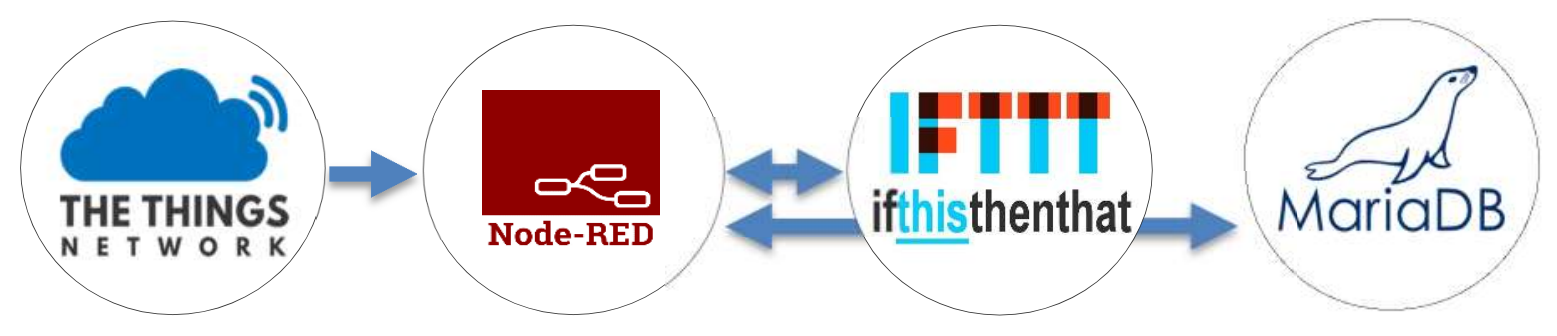

Figure 8 - System primary applications

\section{Management System}

This project features consist of user-related features such as indicators, charts or interactive buttons, to perform actions over devices, and automatic features such as timers and sensor-based rules.

Most indicators provide the user with a quick and easy way to see the current state of a classroom, for example, if the light is on and the room is empty, or if the room is too cold and the air conditioning is off. Each column on the dashboard (Figure 9), represents a single room with all the sensors values and other values such as current air conditioning state, daily air conditioning working time and current light state. At a secondary window on the dashboard, all variables are displayed inline charts with a 12 hour time period. 
As part of the energy management system integration, the available API was used to retrieve realtime data (Figure 10), more accurate than the solution provided with the system because the original website is limited to a minimum real-time consumption of $100 \mathrm{~W}$ which reduces this tool capabilities.

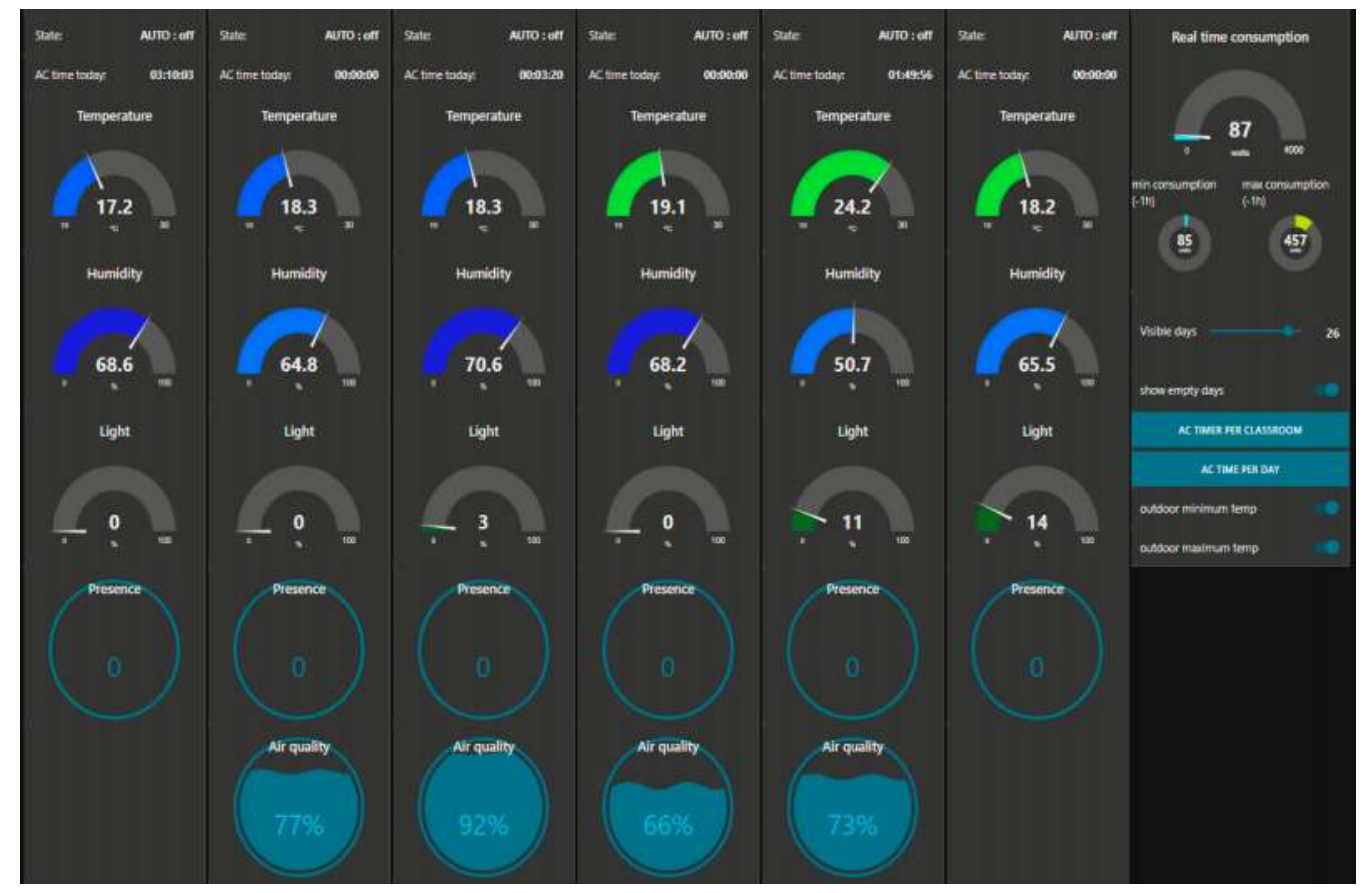

Figure 9 - Node-RED Dashboard, indicators and real time consumption view

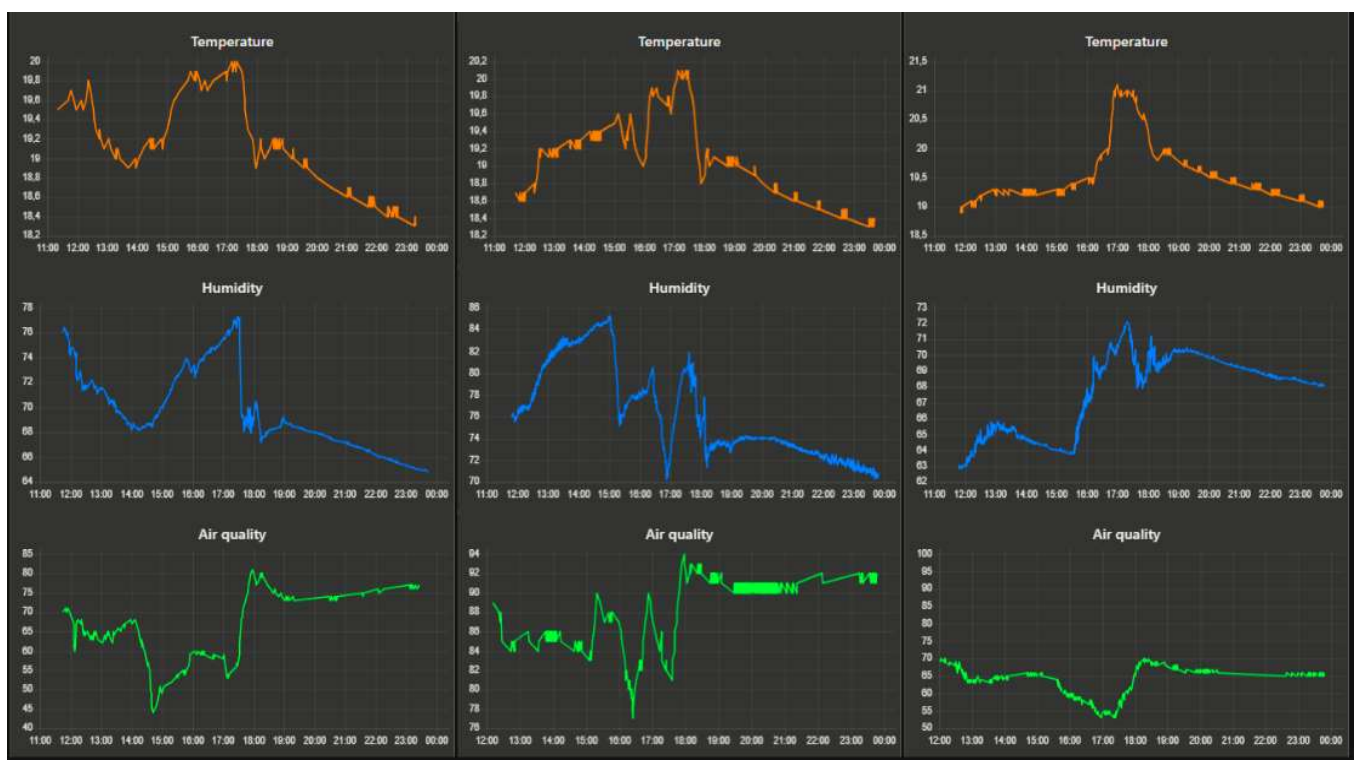

Figure 10 - Node-RED Dashboard, charts view

An energy management tab presents indicators of the minimum and maximum recorded consumption values within the last hour and the last 24h, (Figure 11). This feature was requested by the school owners as a preventing measure for unwanted energy waste, especially during weekends 
A notification email is sent to specific employees when an abnormal consumption is detected during non-work days or during the night, for which no automation rule is possible.

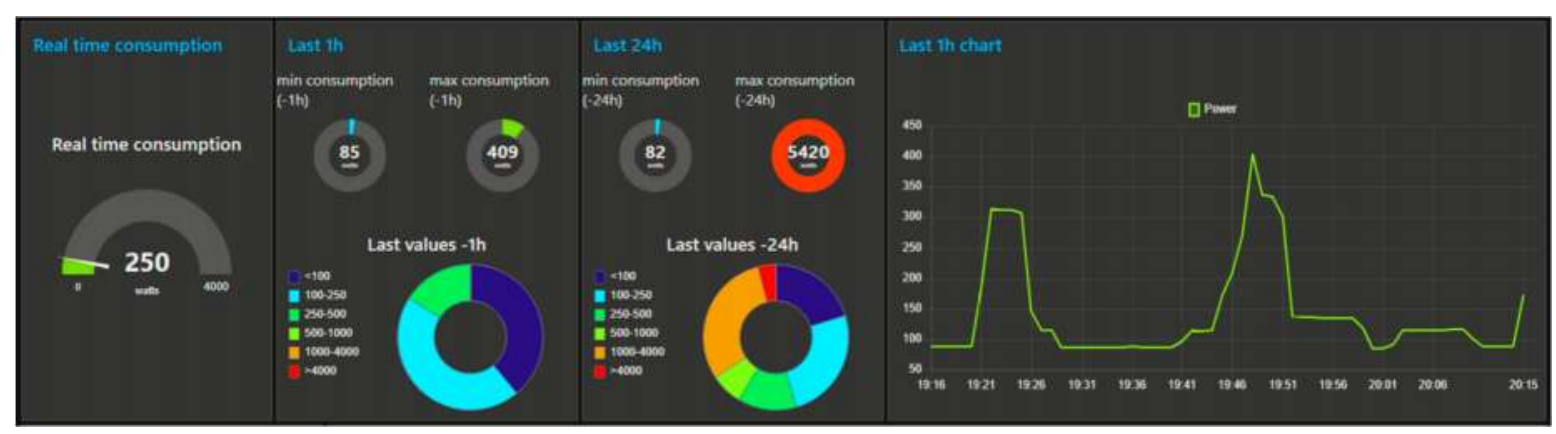

Figure 11 - Node-RED Dashboard, Real time, last 1 h and last 24 h consumption indicators

Air conditioning systems are one of the biggest energy consumers on the building, a dedicated bars chart (Figure 12) shows the time each room's A/C was on allowing the user to understand which rooms require more heating or cooling per day. It's also possible to compare the total amount of time of all $\mathrm{A} / \mathrm{C}^{\prime} \mathrm{s}$ together, with the maximum and minimum expected temperatures for the District, and understand how outdoor temperatures and general weather affects indoor temperatures and humidities and also daily consumptions (Figure 13).

The last chart, (Figure 14 and 15), shows a daily view of energy consumptions by 4 different modes, linear consumption, accumulated consumption, useful for detecting the period of the day when consumptions increases or decreases relative to other days, and minimum and maximum consumption value recorded each hour, particularly useful for detecting anomalies and power limits.

Knowing the maximum power usage per hour, it is possible to determine the necessary contracted power that also represents part of the energy bill.

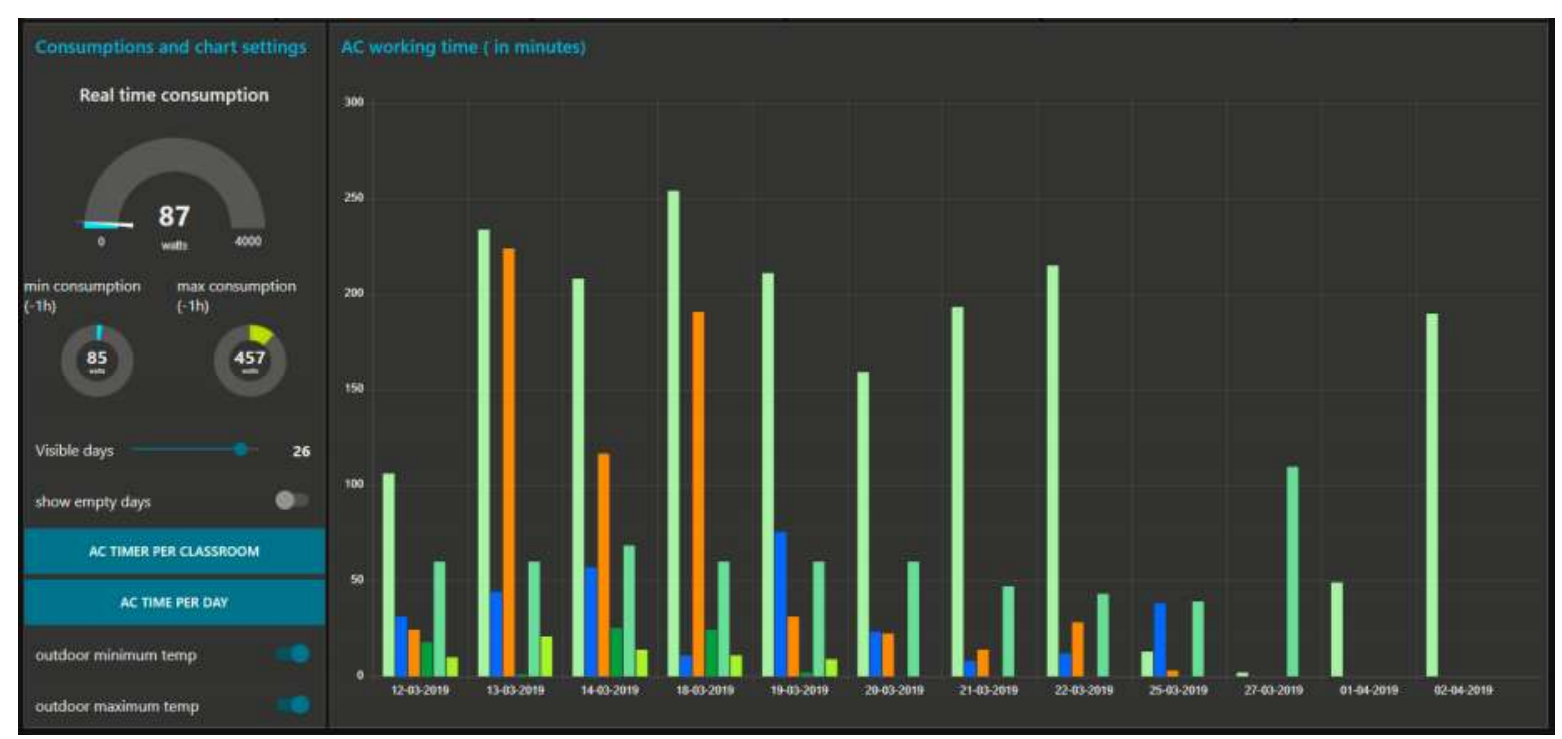

Figure 12 - Node-RED Dashboard, AC usage time in minutes per classroom per day 


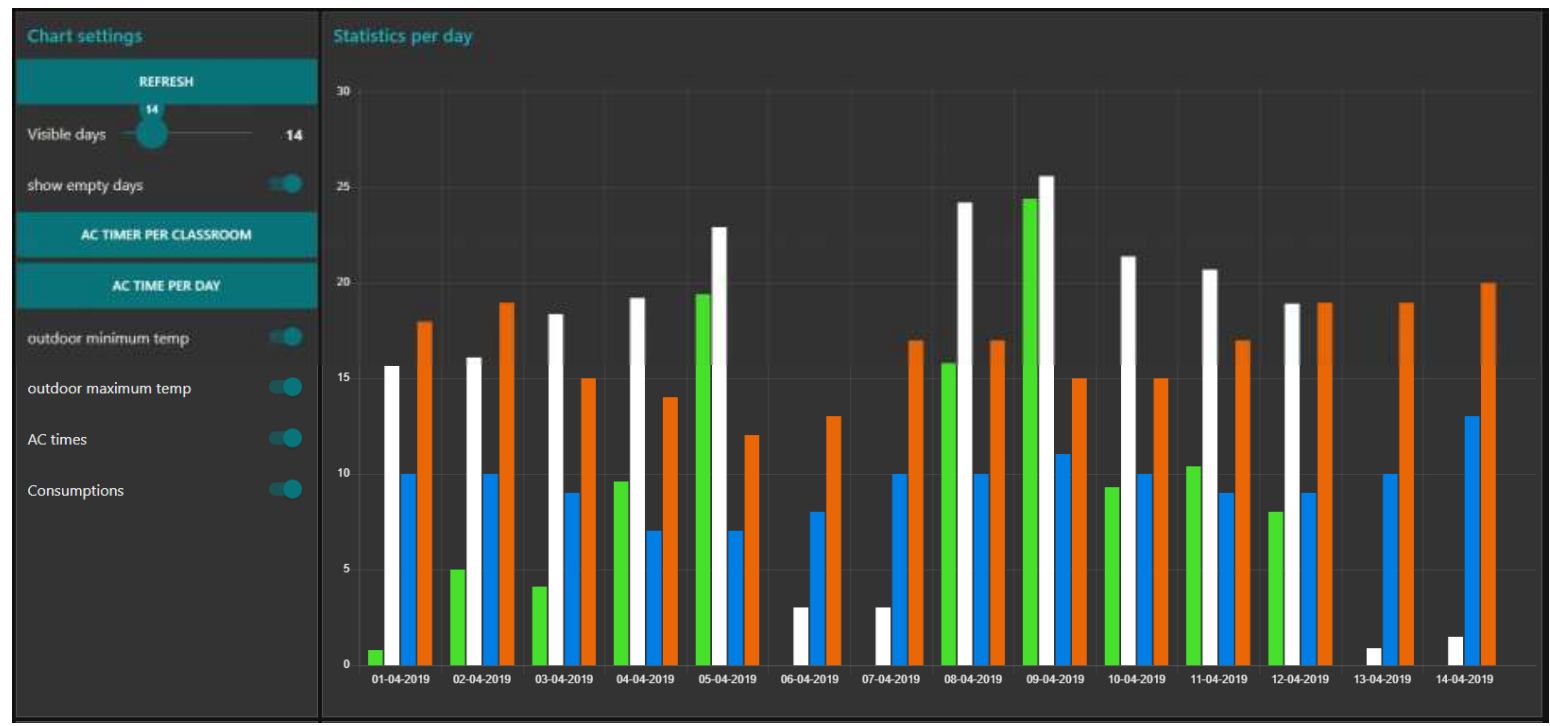

Figure 13 - Node-RED Dashboard, AC usage time in hours vs outdoor temperatures vs daily energy consumption

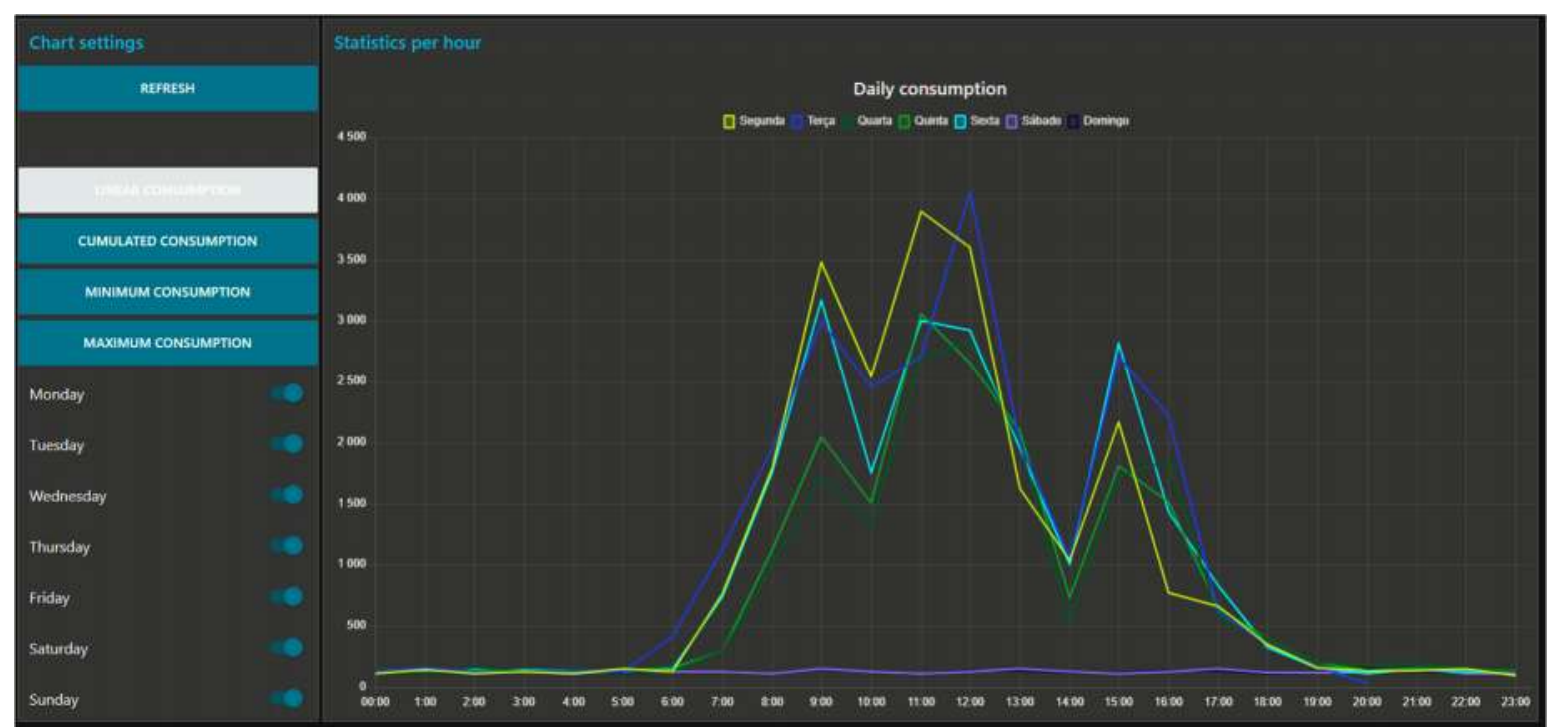

Figure 14- Node-RED Dashboard, daily linear consumption

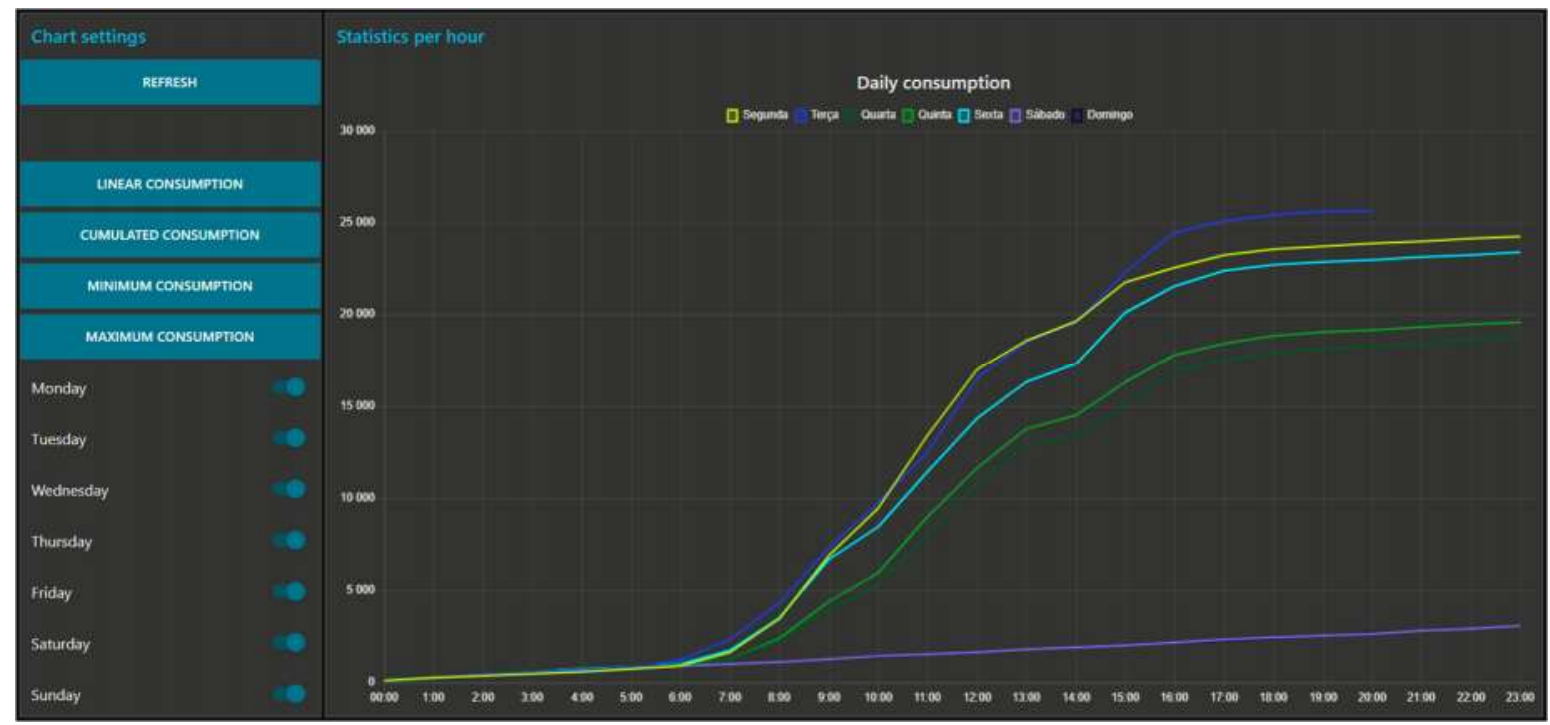

Figure 15 - Node-RED Dashboard, daily cumulated consumption 
The dashboard interface, as mentioned above, includes some interactive buttons and controllers, mainly for $\mathrm{A} / \mathrm{C}$ and lighting control purposes. The $\mathrm{A} / \mathrm{C}^{\prime} \mathrm{s}$ of each room can be turned On or Off from the dashboard remotely for 1 hour, or for the rest of the day, however, the default mode is AUTO, meaning, autonomous control based on sensors readings. The user can define a minimum and maximum temperature for the room to be used with the automatic rules. Ceiling lights can also be turned on or off from the dashboard, using the WiFi switches in Figure 16 as a bridge between the internet and the circuit itself.

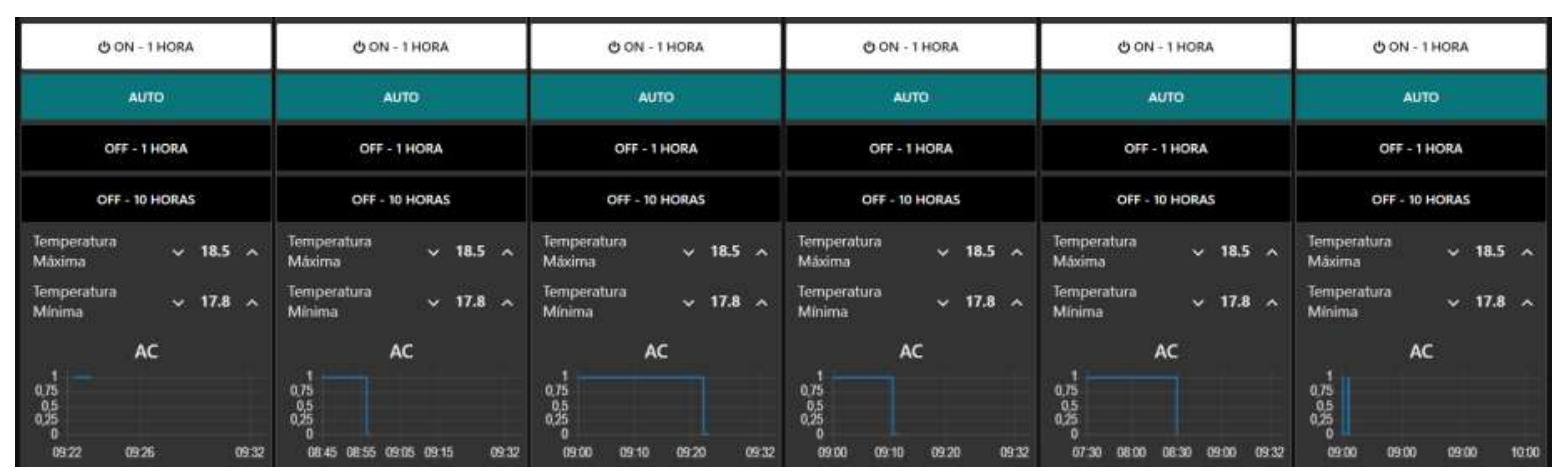

Figure 16 - Node-RED Dashboard, AC controllers

For simplification purposes, the system description is only described for the Winter period.

Running on AUTO mode, each AC works following a list of pre-set rules and priorities. Manual override is considered the highest priority, followed by the maximum Temperature value defined so that when the room is at a desirable temperature, there is no need to turn on the A/C. A weekly schedule determines whether it should be On or Off during each hour of the week, then a daily timer overrides the presence sensor for determined periods of the day when the $\mathrm{A} / \mathrm{C}$ should be on but no presence is detected, meaning sleep hours. Finally, if the room is below minimum Temperature, the weekly schedule indicates an On state, and the presence sensor is active, the A/C is turned on until the required temperature is reached.

The following flowchart, Figure 17, provides a simplified view of how the automation rules work.

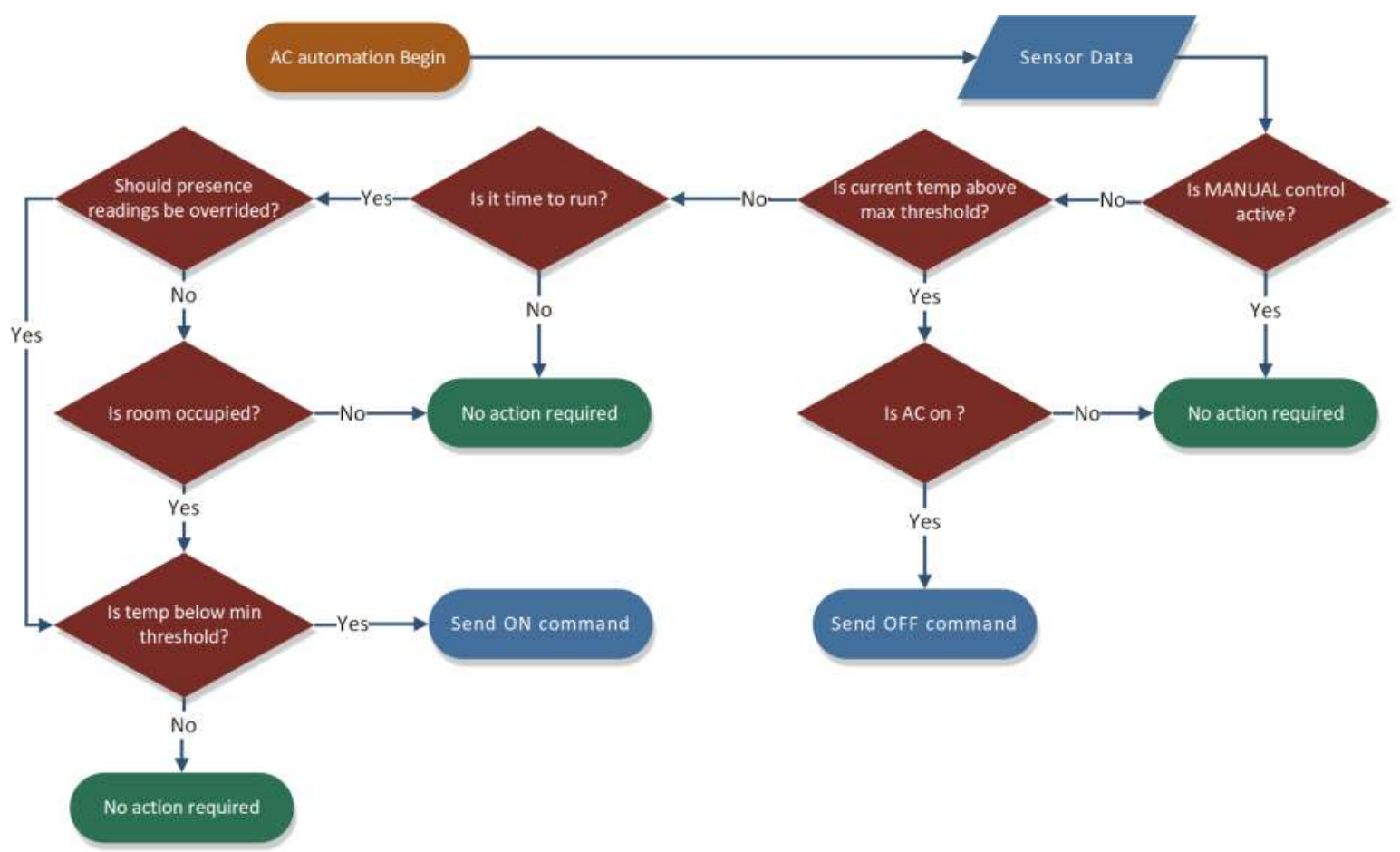

Figure 17 - AC automation rules schematized on a flowchart 
If Humidity values reach above $70 \%$, a dehumidification command is triggered and sent to $\mathrm{A} / \mathrm{C}$ units, so that exceeding moisture is removed from the air.

For the ceiling lights, the light sensors are able to distinguish between natural light and artificial light, so that the system can evaluate if the natural light is enough. After some calibration and position adjustments on the circuit board, the following automation rules were implemented:

- Such as the AC automation rules, the manual override has the highest priority, followed by the weekly schedule dedicated to lighting purposes.

- If the difference between 2 consecutive light values is above a determined percentage, it means the light has been turned On, and if the oldest light value was considered enough, the light is forced to be turned off.

- When the light is On, a presence-based rule compares the last 2 values and if both show no presence, the light is turned Off.

- When the light is on but the room is occupied, if the value corresponds to the artificial light plus a determined margin, it means that natural light is enough and the light is turned off.

A variety of notification alarms were also set for lights and $\mathrm{A} / \mathrm{C}^{\prime} \mathrm{s}$ turned On when no presence is detected at the school.

Air quality is mostly used as a need indicator of air renewal and for parent's knowledge, however, equipping each room's ionic air filter with a smart power socket (Figure 5), allows them to be turned on when the air quality is too low. Air quality statistics can also be sent to parent's emails or mobile phone Apps by IFTTT email service.

\section{Major Findings}

Before the automation rules were created, all sensors data were observed for a week, to understand how temperature and humidity changed considering the time of the day and outdoor temperatures. As expected, during the night period, temperatures lower around 2-3 degrees Celsius and humidity lower 10-20\%. During labour hours temperature would quickly rise in the morning when the $\mathrm{A} / \mathrm{C}^{\prime}$ s were turned on, then remain constant until closing hours when it begins to drop after the $\mathrm{A} / \mathrm{C}^{\prime} \mathrm{s}$ were turned off. To keep a constant temperature on each room during the day, air conditioning systems remain On till manually turned off, and despite the pre-set temperature on the remote, the real room temperature is 1 to 2 degrees above, which proves the inefficiency of standard A/C's AUTO mode.

After the automation rules were implemented, the temperature behaviour altered completely, changing from constant to a saw line type, (Figure 18 and 19), this is due to the established maximum and minimum temperatures, that regulate each room's temperature according to these two limits. Besides limiting the temperature to a certain range, the system also prevents unnecessary usage when the room is empty and because the amount of time it remains off is significant, the energy saved is reflected on the daily consumption.

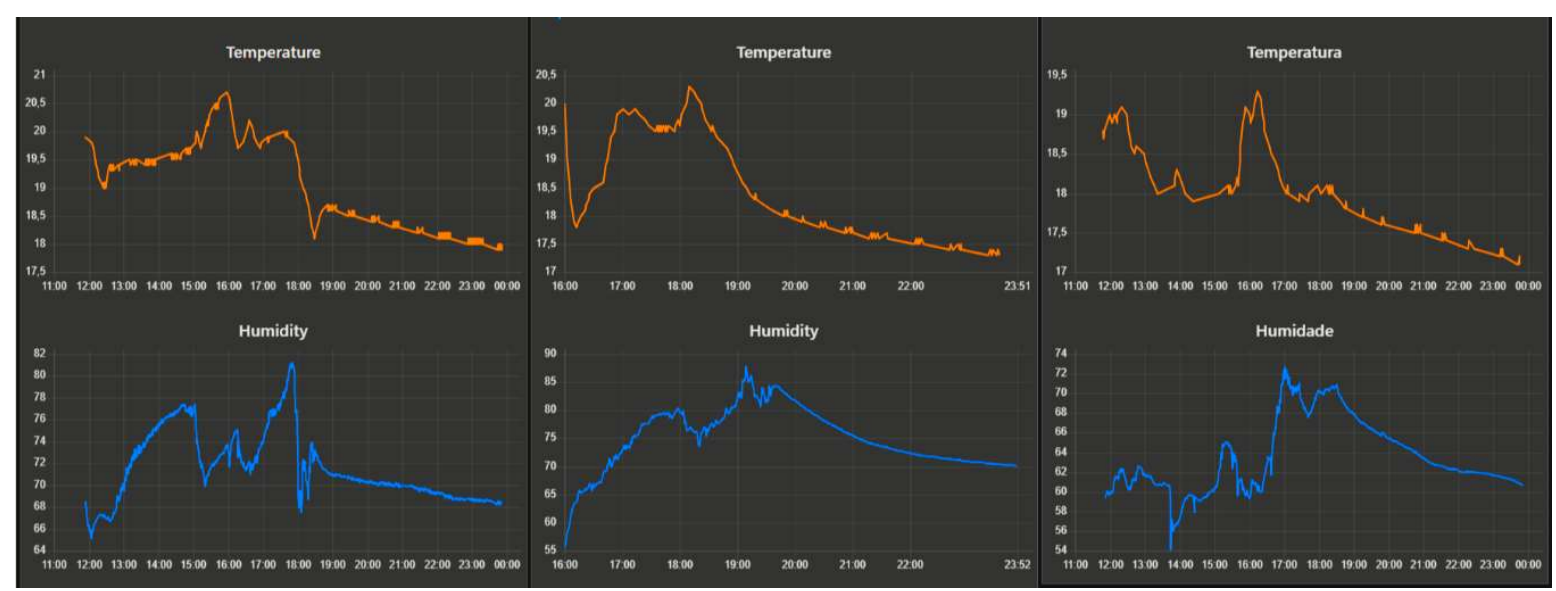

Figure 18-Node-RED Dashboard, temperature variation overnight period 


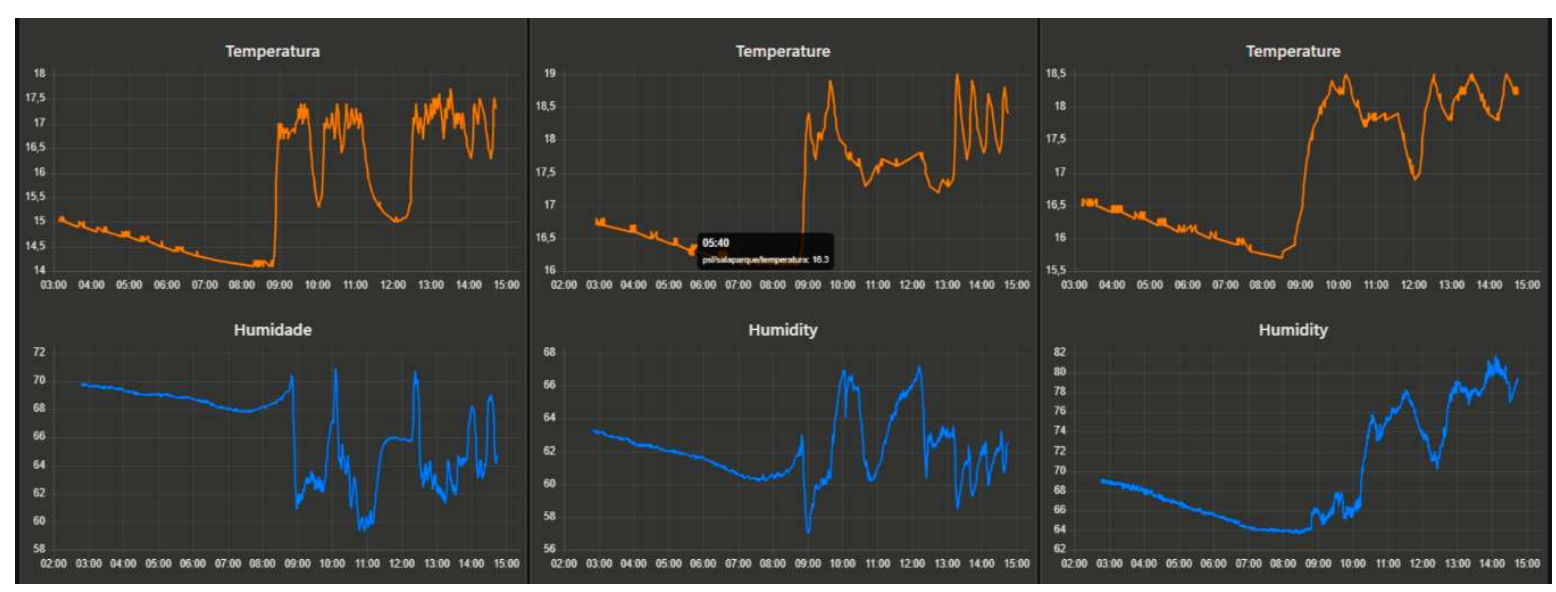

Figure 19 - Node-RED Dashboard, temperature variation during system operation

The amount of time each room takes to lower 1 degree after the $\mathrm{A} / \mathrm{C}$ is turned Off, is directly proportional to the room size and inversely proportional to the room capacity during the day, meaning that a smaller room with fewer students cools down much faster and requires more heating and more often. This is perceived on the next graph, (Figure 20), where the first column of each day representing the same room, has more working time then all the others recurrently.

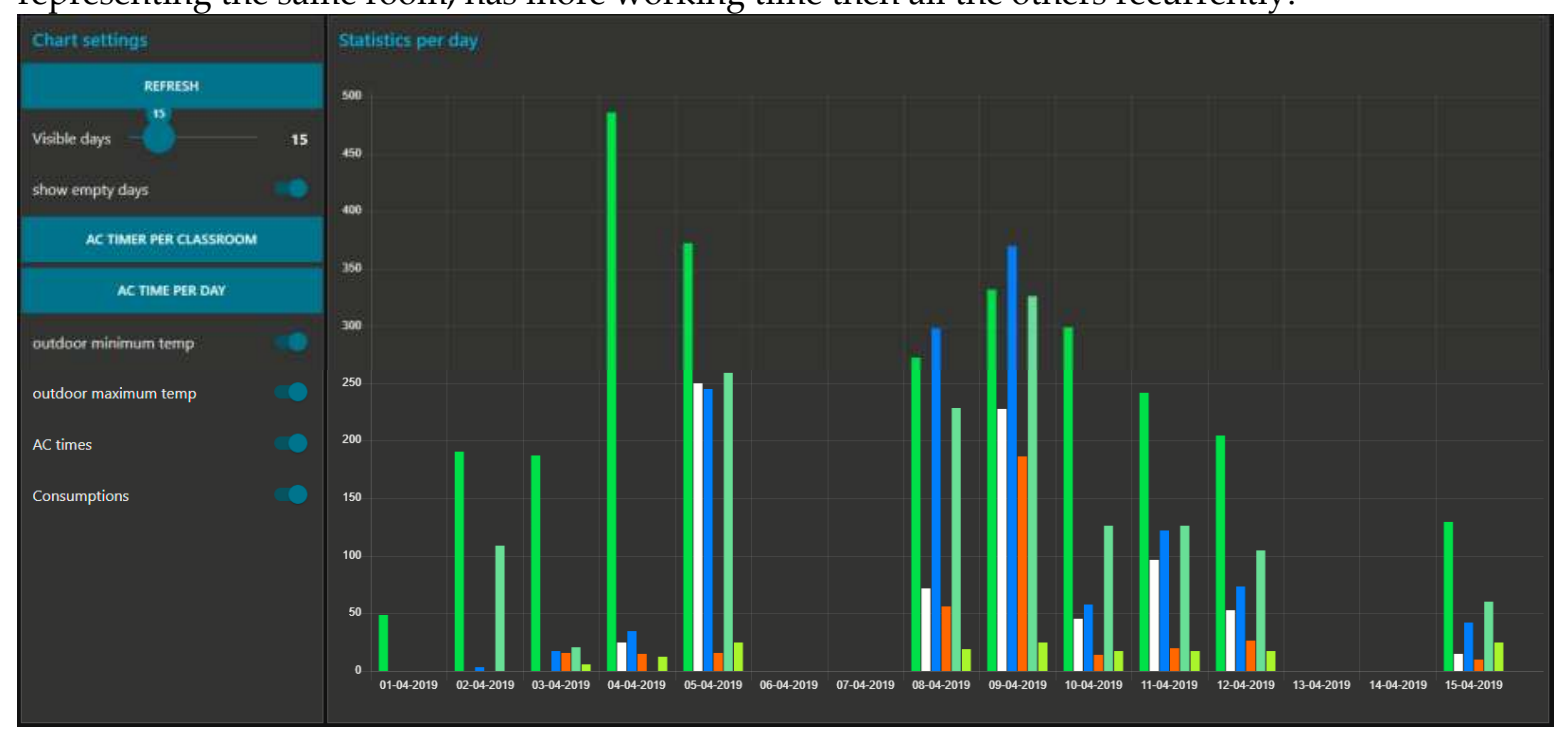

Figure 20 - Node-RED Dashboard, AC working time per room

An anomaly detected by interpretation of system data allowed to discover defective window isolation when during a rainy night, when all room's humidity was getting lower, the humidity on a single classroom was showing the opposite behaviour and getting higher.

The room that presented the fastest temperature drop rate, on a windy day, begun to display unusual $\mathrm{A} / \mathrm{C}$ working times, since it worked for hours straight without stopping, after a detailed analysis of the room, it was discovered a pipe inside a decommissioned energy circuit board cabinet that connected to the high ceiling where temperatures are much lower and much less isolation, causing the cold air from the street to flow down the pipe into the room.

Besides the anomalies mentioned above, another anomaly was detected multiple times, related to strange consumption values during the night or weekends, such as the thermal accumulator that heats up the water every morning, started to work at a weekend, because the timer was unintentionally changed (Figure 21 and 22), or one of the fridges that were working nonstop because the door wasn't properly closed or the temperature was set to MAX.

Below is an example of the fridge consumption pattern, marked with circles, and the thermal accumulator pattern from 6:45 to 8:45 am. 


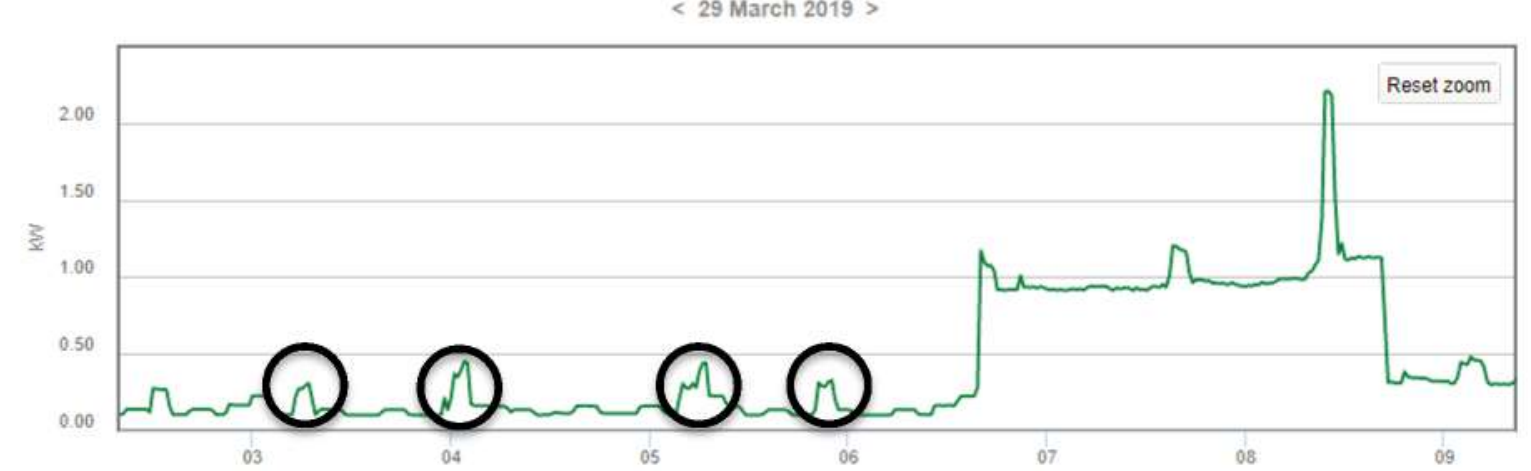

Figure 21 - Energy management system dashboard, fridge and thermal accumulator signatures

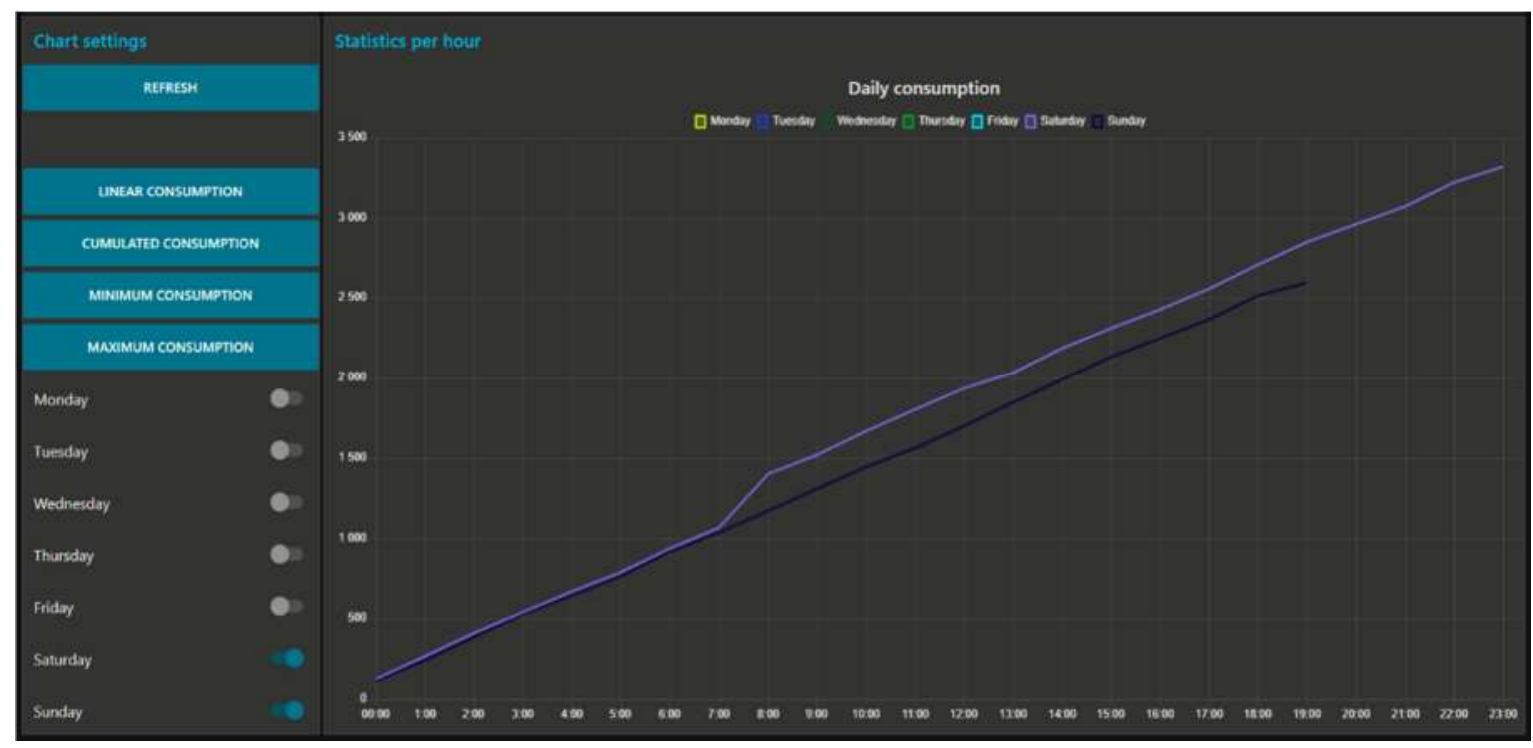

Figure 22 - Node-RED Dashboard, cumulative consumption with and without the thermal accumulator anomaly

An experiment relating $\mathrm{A} / \mathrm{C}$ working time and energy consumption was carried out by changing the minimum and maximum temperature limit of every classroom for a day. On the first day (Monday) the temperatures were set to default because Monday is usually when more energy is spent due to the building lower temperatures after the weekend when the school is empty and no Air conditioning is used. On Tuesday, both limits were increased 1 degree and on Wednesday, default values were restored. The used parameters can be consulted on the next table (Figure 23), followed by the results.

\begin{tabular}{|c|c|c|c|c|}
\hline Day of the week & $\begin{array}{c}\text { Maximum } \\
\text { Temperature }\end{array}$ & $\begin{array}{c}\text { Minimum } \\
\text { Temperature }\end{array}$ & $\begin{array}{c}\text { AC working } \\
\text { Time }\end{array}$ & $\begin{array}{c}\text { Energy } \\
\text { Consumption }\end{array}$ \\
\hline $\begin{array}{c}\text { Monday } \\
(08 / 04 / 2019)\end{array}$ & $18,5^{\circ} \mathrm{C}$ & $17,8^{\circ} \mathrm{C}$ & 15,8 hours & $24,21 \mathrm{KWh}$ \\
\hline $\begin{array}{c}\text { Tuesday } \\
(09 / 04 / 2019)\end{array}$ & $19,5^{\circ} \mathrm{C}$ & $18,8^{\circ} \mathrm{C}$ & 24,4 hours & $25,6 \mathrm{KWh}$ \\
\hline $\begin{array}{c}\text { Wednesday } \\
(10 / 04 / 2019)\end{array}$ & $18,5^{\circ} \mathrm{C}$ & $17,8^{\circ} \mathrm{C}$ & 9,3 hours & $21,4 \mathrm{KWh}$ \\
\hline \multicolumn{2}{|r|}{ Figure 23-Experiment variables and results } \\
\hline
\end{tabular}

Figure 23 - Experiment variables and results 
On Monday, April $8^{\text {th }}$, air conditioning units worked 15,8 hoursaltogether, because of the number of hours without thermal transferences other than outdoor to indoor. On Tuesday, April $9^{\text {th }}$ when each room limits were increased by $1^{\circ} \mathrm{C}$, the total amount of $\mathrm{A} / \mathrm{C}$ working time increased by $50 \%$, and energy consumption increased 1,5 KWh. It was on Wednesday, April $10^{\text {th }}$, that a bigger difference was noticed, having A/C units working 62\% less time and energy consumption dropping 4,2 KWh. Such an energy usage reduction results in approximately $23 €$ of savings every month for a school with 6 classrooms. All the previous data was collected from the dashboard (Figure 24).

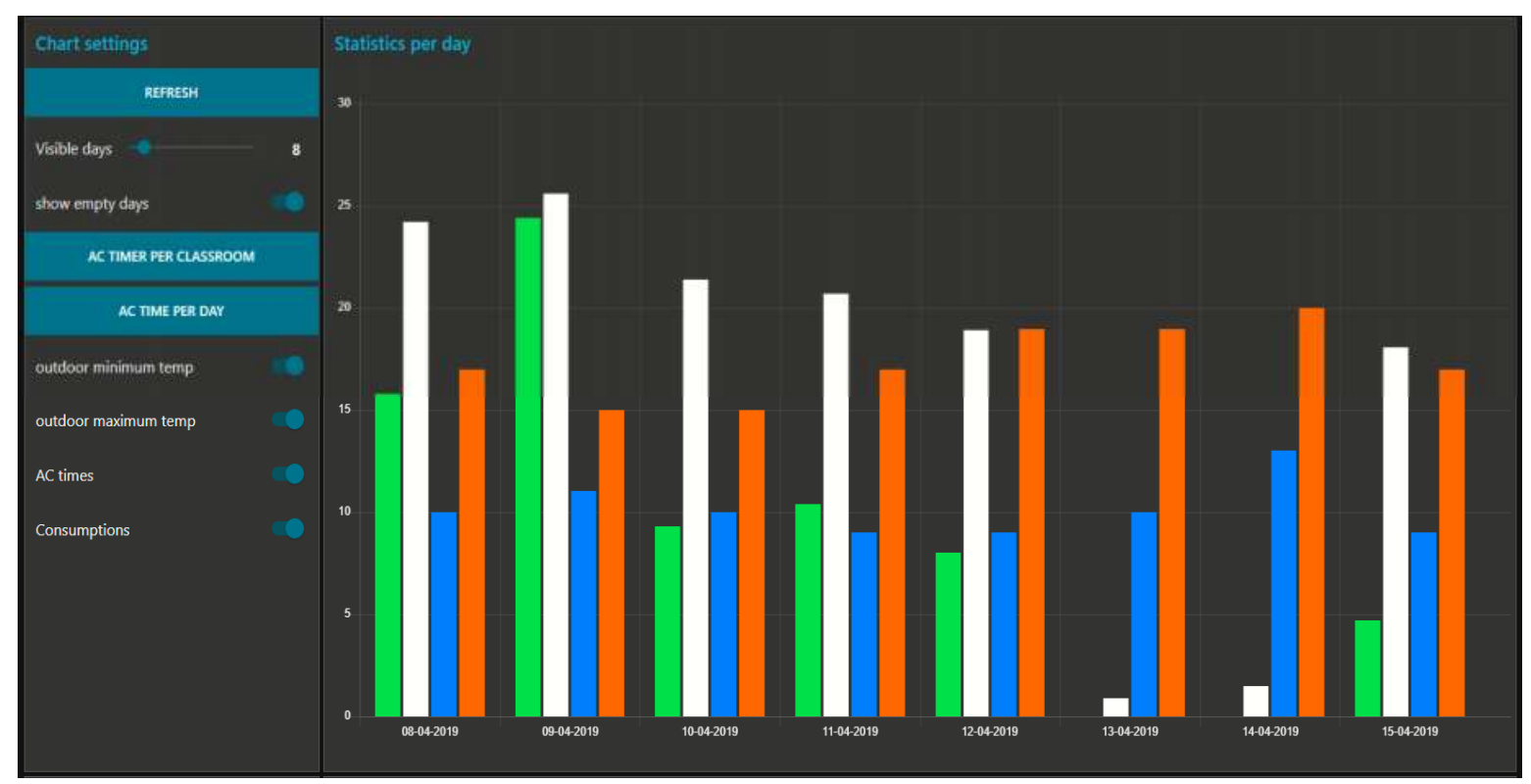

Figure 24 - Node-RED Dashboard, experiment results with AC working time, daily energy consumption and max / min outdoor temperatures

Using light sensors data is possible to determine when the artificial light is turned on and when it isn't. On the next Figure 25, it is possible to observe the behaviour of the light sensor and relate it to a light state in two classrooms. When a percentage difference of more than $10 \%$ is detected on 2 consecutive readings, it means the light was turned On or Off. After testing, it was concluded that $50 \%$ of the maximum light percentage is enough for working purposes, meaning that when the light is turned on, and the previous value equals more than $50 \%$ of the maximum value the light is automatically turned off.
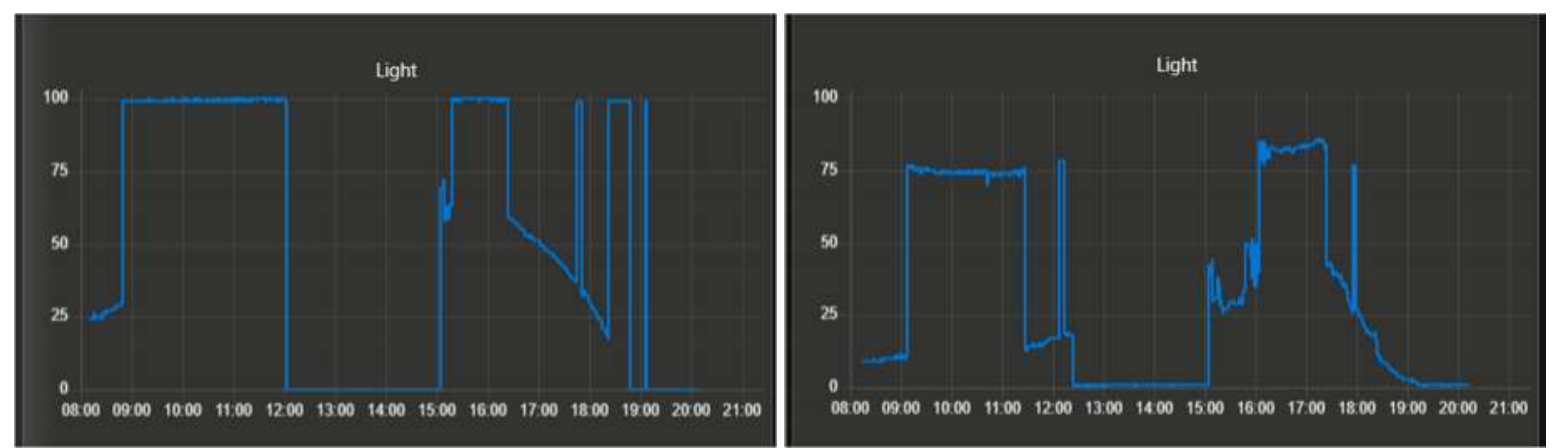

Figure 25 - Node-RED Dashboard, light charts during labor hours

Air quality presents a direct relation with room occupancy and events such as opening a window or closing the room for sleep hours. On the next Figure 26, it is possible to identify a window opening event at 17:30 on the left chart relating to a small size classroom, where air quality changes faster, and the chart on the right, relating to a large size classroom where occupation is the most relevant variable. For certain periods of the day, if Air quality reaches below $40 \%$, ionic filters are activated till air quality returns to acceptable values (> 70\%). 


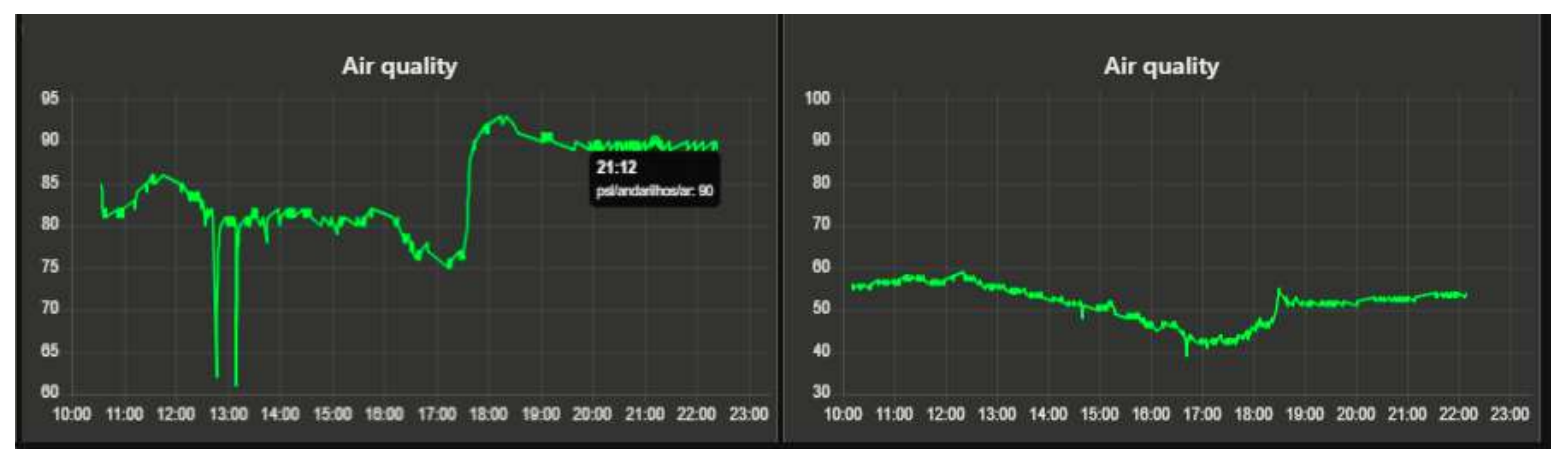

Figure 26 - Node-RED Dashboard, Air quality charts for 2 different rooms

\section{Energy Savings}

Since 2016 when the energy monitoring system was implemented, and daily energy consumption during Winter was on average $33 \mathrm{KWh}$, and during Summer, $20 \mathrm{KWh}$, the knowledge about the consumptions of the building allowed us to better understand the pattern and associate it with electric equipment who are responsible for most of the energy bill. Energy saving policies were established and allowed a significant reduction in power usage and costs for the first months.

This reduction was followed by a stability period of time when it was apparently impossible to reduce even more, however, as soon as the LoRa system was created another significant power consumption reduction took place.

As previously mentioned, the data analysis concluded that Kitchen appliances such as fridges or the dishwasher, and Air Conditioning systems were the main power consumers in the building, however with more precise data, the Node-RED dashboard, allowed us to discover other cases energy was being wasted. Doing an experiment with each circuit breaker at the main electrical switchboard the following standby power was detected:

- 3 circuit breakers power an Air conditioning unit each, and each of them has a persistent energy waste of 30 watts even with all units Off, which means 90 watts total;

- Emergency lights have a persistent power consumption of 50 watts;

- Kitchen appliances such as the mosquito net spend 60 watts;

- Each computer + monitor spends 5 watts even if completely Off;

All the referred power usage is considered waste during non-labour days, non-labour hours, which represents $50 \%$ of the day or even more when there is no use during labour hours.

Using smart WiFi power sockets and a WiFi power socket for energy circuits, on each of these devices, and scheduling On and Off timers, it's possible to save more than $3 \mathrm{KWh}$ per day, which at the current energy price is equivalent to approximately $364 €$ per year, more than 3 regular months of energy cost.

\section{Conclusions}

In 2016 the system was implemented and successive improvements were performed. Lora communication allows battery sensors to be placed across the building without the requirement of a power outlet or a power source replacement for a few years, and continuously provide reliable data, essential to apply sustainable and efficient measures. Since the project began, more than 750000 sensor readings were taken and stored, being 150000 of the temperature readings. AC units were turned on and off 3060 times when their usage was required or not, and more than $130 \mathrm{kWh}$ were saved, based on historical data. Besides all the improvements the system provided, both students and employees sense of comfort has increased.

Major work contribution is a LoRa IoT open source solution for building management, where a set of consumption reports are available in real-time for management savings. Several what-if scenarios can be performed for automatic savings actions. Also, a real-time notification can be performed for email, SMS or mobile device App to alert for unexpected behaviour. This approach 
can be applied to any building from houses to companies and campus, provide a monitor control environment. In this IoT, Low Power and Long Range communication, like LoRa play an important role since sensors can be easily installed without cablings using internal batteries.

An interaction approach to air condition or heating systems was developed based on an infrared approach, that can be applied to a diversity of equipment. We data collected is manipulated to create an integrated view in dashboards that can be sent to mobile devices.

This information visualized and correspondent actions in these three years conduct to save around $20 \%$ to $25 \%$ and investments were paired in the first year of operation.

Author Contributions: B.M. is a Master student that performed all development work. J.C.F. is thesis supervisor and organized all work in the computer science subject, and N.C. revised LoRa connections and performed a major work revision.

Funding: This work has been partially supported by Portuguese National funds through FITEC programa Interface, with reference CIT "INOV - INESC INOVAÇÃO - Financiamento Base".

\section{References}

1. IEA - Transition to Sustainable Buildings: Strategies and Opportunities to 2050 (2013) (Available from: http://www.iea.org/etp/buildings/)

2. Xiaodong Cao, Xilei Dai, Junjie Liu, Building energy-consumption status worldwide and the state-ofthe-art technologies for zero-energy buildings during the past decade, Energy and Buildings, Volume 128, 2016, Pages 198-213, ISSN 0378-7788, https://doi.org/10.1016/j.enbuild.2016.06.089.

3. Meola, A. (2016, September 16). How IoT in Education is Changing the Way We Learn.

4. Asseo, I., Johnson, M., Nilsson, B., Chalapathy, N., \& Costello, T. J. The Internet of Things: Riding the Wave in Higher Education. (2016, June 27).

5. Report available at https://www.mckinsey.com/business-functions/digital-mckinsey/our-insights/theinternet-of-things-the-value-of-digitizing-the-physical-world

6. Report available at https://www.enertiv.com/resources/blog/4-limitations-bms-data

7. João C. Ferreira, Vítor Monteiro, Jose Afonso and João L. Afonso (2018). An energy management platform for public buildings. Electronics 2018, 7(11), 294; https://doi.org/10.3390/electronics7110294

8. João C. Ferreira and Ana L. Martins, (2018). Building a community of users for open market energy. Energies 2018, 11(9), 2330; https://doi.org/10.3390/en11092330

9. M. Abo-Zahhad, S.M. Ahmed, M. Farrag, M.F.A. Ahmed, A. Ali, "Design and implementation of building energy monitoring and management system based on wireless sensor networks", 2015 Tenth International Conference on Computer Engineering \& Systems (ICCES), pp. 230-233, 2005.

10. N.H. Nguyen, Q.T. Tran, J.M. Leger, T.P. Vuong, "A real-time control using wireless sensor network for intelligent energy management system in buildings", 2010 IEEE Workshop on Environmental Energy and Structural Monitoring Systems, pp. 87-92, 2010.

11. J. Byun, I. Hong, B. Kang, S. Park, "Implementation of an Adaptive Intelligent Home Energy Management System Using a Wireless Ad-Hoc and Sensor Network in Pervasive Environments", 2011 Proceedings of 20th International Conference on Computer Communications and Networks (ICCCN), pp. 1-6, 2011.

12. J. Han, C.S. Choi, W. Choi, K. Park, I. Lee, S.H. Kim, "Smart home energy management system including renewable energy based on ZigBee and PLC", IEEE Trans. Consumer Electron, vol. 60, no. 2, pp. 198-202, May 2014.

13. J. Wang, J. Huang, W. Chen, J. Liu, D. Xu, "Design of IoT-based energy efficiency management system for building ceramics production line", 2016 IEEE 11th Conference on Industrial Electronics and Applications (ICIEA), pp. 912-917, 2016.

14. G. Mingming, S. Liangshan, H. Xiaowei, S. Qingwei, "The System of Wireless Smart House Based on GSM and ZigBee", 2010 International Conference on Intelligent Computation Technology and Automation, pp. 1017-1020, 2010.

15. J. Serra, D. Pubill, A. Antonopoulos, C. Verikoukis, "Smart HVAC control in IoT: Energy consumption minimization with user comfort constraints", The Scientific World Journal, pp. 1-11, 2014.

16. K.F. Fong, V.I. Hanby, T.T. Chow, "HVAC system optimization for energy management by evolutionary programming", Energy and Buildings, vol. 38, no. 3, pp. 220-231, 2006. 
17. Tacklim Lee, Seonki Jeon, Dongjun Kang, Lee Won Park, Sehyun Park, "Design and implementation of intelligent HVAC system based on IoT and Bigdata platform", 2017 IEEE International Conference on Consumer Electronics (ICCE), pp. 398-399, 2017.

18. Y.T. Lee, W.H. Hsiao, C.M. Huang, S.C.T. Chou, "An integrated cloud-based smart home management system with community hierarchy", IEEE Trans. Consumer Electron, vol. 62, no. 1, pp. 1-9, Feb. 2016.

19. E. Rodriguez-Diaz, J.C. Vasquez, J.M. Guerrero, "Intelligent DC Homes in Future Sustainable Energy Systems: When efficiency and intelligence work together", IEEE Consumer Electron. Magazine, vol. 5, no. 1, pp. 74-80, Jan. 2016.

20. N. Kushiro, S. Suzuki, M. Nakata, H. Takahara, M. Inoue, "Integrated residential gateway controller for home energy management system", IEEE Transactions on Consumer Electronics, vol. 49, no. 3, pp. 629-636, Aug. 2003.

21. M. Erol-Kantarci, H.T. Mouftah, "Wireless Sensor Networks for Cost-Efficient Residential Energy Management in the Smart Grid", IEEE Transactions on Smart Grid, vol. 2, no. 2, pp. 314-325, June 2011.

22. K. Dittawit, F.A. Aagesen, "Home energy management system for electricity cost savings and comfort preservation", 2014 IEEE Fourth International Conference on Consumer Electronics Berlin (ICCEBerlin), pp. 309-313, 2014. 